PROCEEDINGS OF THE

AMERICAN MATHEMATICAL SOCIETY

Volume 138, Number 3, March 2010, Pages 1113-1120

S 0002-9939(09)10123-5

Article electronically published on October 26, 2009

\title{
THE SPAN FOR HAUSDORFF CONTINUA
}

\author{
ANTONIO PELÁEZ
}

(Communicated by Alexander N. Dranishnikov)

\begin{abstract}
The author previously defined the surjective semispan for Hausdorff continua and he proved that chainable continua have empty surjective semispan. In this paper, we define the semispan, the surjective span and the span of a Hausdorff continuum. We characterize the emptiness of these notions in terms of universal mappings to prove that a continuum has empty span (semispan) if and only if each of its subcontinua has empty surjective span (semispan). We also prove that the emptiness of these notions is invariant under inverse limits.
\end{abstract}

\section{Definitions And notations}

Given the relations $U$ and $V$ on a set $X$, the inverse relation of $U$ is the set:

$$
-U=\{(y, x):(x, y) \in U\},
$$

and the composition of $U$ and $V$ is the set:

$$
U+V=\{(x, z): \text { there exists a } y \in X \text { such that }(x, y) \in U \text { and }(y, z) \in V\} .
$$

We also write $1 V=V$ and, for a positive integer $n,(n+1) V=n V+V$.

The diagonal of $X$ is the set $\Delta=\{(x, x): x \in X\}$. An entourage of the diagonal is a set $V \subset X \times X$ containing $\Delta$ such that $V=-V$; the family of all entourages of the diagonal is denoted by $\mathcal{D}_{X}$. If we have $x, y \in X$ and $V \in \mathcal{D}_{X}$ such that $(x, y) \in V$, then we say that the distance between $x$ and $y$ is less than $V$ and we write $|x-y|<V$; otherwise we write $|x-y| \geq V$. If for every pair of points $x, y$ of a set $A \subseteq X$ and $V \in \mathcal{D}_{X}$ we have that $|x-y|<V$, i.e., if $A \times A \subseteq V$, we say that the diameter of $A$ is less than $V$ and we write $\delta(A)<V$; otherwise we write $\delta(A) \geq V$.

Given a point $x \in X$ and $V \in \mathcal{D}_{X}$, the ball with center $x$ and radius $V$ (briefly, the $V$-ball about $x)$ is the set $B(x, V)=\{y \in X:|x-y|<V\}$. For a set $A \subseteq X$ and a $V \in \mathcal{D}_{X}$, the $V$-ball about $A$ is the set $B(A, V)=\bigcup\{B(x, V): x \in A\}$.

A uniformity on a set $X$ is a subfamily $\mathcal{U}$ of $\mathcal{D}_{X}$ such that:

U1. If $V \in \mathcal{U}$ and $V \subseteq W \in \mathcal{D}_{X}$, then $W \in \mathcal{U}$.

U2. If $V, W \in \mathcal{U}$, then $V \cap W \in \mathcal{U}$.

U3. For every $V \in \mathcal{U}$ there exists $W \in \mathcal{U}$ such that $2 W \subseteq V$.

$\mathrm{U} 4 . \cap \mathcal{U}=\Delta$.

Received by the editors December 5, 2008, and, in revised form, April 16, 2009.

2000 Mathematics Subject Classification. Primary 54F15; Secondary 54H25, 54E15.

Key words and phrases. Hausdorff continua, span, surjective span, semispan, surjective semispan.

(C)2009 American Mathematical Society Reverts to public domain 28 years from publication 
A family $\mathcal{B} \subseteq \mathcal{U}$ is a base for the uniformity $\mathcal{U}$ if for every $V \in \mathcal{U}$ there exists $W \in \mathcal{B}$ such that $W \subseteq V$. A uniform space is a pair $(X, \mathcal{U})$, where $\mathcal{U}$ is a uniformity on the set $X$.

Theorem 1.1 ([1, 8.1.1]). For every uniformity $\mathcal{U}$ on a set $X$ the family

$$
\mathcal{O}=\{G \subseteq X \text { : for every } x \in G \text { there exists a } V \in \mathcal{U} \text { such that } B(x, V) \subseteq G\}
$$

is a topology on $X$ and the topological space $(X, \mathcal{O})$ is a $T_{1}$-space.

The topology $\mathcal{O}$ is called the topology induced by the uniformity $\mathcal{U}$.

If $X$ is a topological space and its topology is induced by a uniformity $\mathcal{U}$, we say that $\mathcal{U}$ is a uniformity on the space $X$.

In fact, the space $(X, \mathcal{O})$, constructed in Theorem 1.1, is a Tychonoff space and:

Theorem 1.2 ([1, 8.1.20]). The topology of a space $X$ can be induced by a uniformity on the set $X$ if and only if $X$ is a Tychonoff space.

Theorem 1.3 ([1, 8.3.13]). For every Hausdorff compact space $X$ there exists exactly one uniformity $\mathcal{U}$ on the set $X$ that induces the original topology of $X$. All entourages of the diagonal $\Delta \subseteq X \times X$ which are open in the Cartesian product $X \times X$ form a base for the uniformity $\mathcal{U}$.

Definition 1.4. A uniform space $(X, \mathcal{U})$ is compact if $X$ with the topology induced by $\mathcal{U}$ is a compact space.

A mapping $f: X \rightarrow Y$ between the uniform spaces $(X, \mathcal{U})$ and $(Y, \mathcal{V})$ is uniformly continuous with respect to the uniformities $\mathcal{U}$ and $\mathcal{V}$ if for every $V \in \mathcal{V}$ there exists $U \in \mathcal{U}$ such that $|f(x)-f(y)|<V$ whenever $|x-y|<U$. In this case we write $f:(X, \mathcal{U}) \rightarrow(Y, \mathcal{V})$. It follows from the definition and Theorem 1.1 that $f$ is a continuous mapping of the space $X$ with the topology induced by $\mathcal{U}$ to the space $Y$ with the topology induced by $\mathcal{V}$.

Theorem 1.5 ([5, 1.8]). Let $\mathcal{U}$ and $\mathcal{V}$ be uniformities on the Hausdorff compact spaces $X$ and $Y$, respectively. A mapping $f: X \rightarrow Y$ is continuous if and only if $f$ is uniformly continuous with respect to the uniformities $\mathcal{U}$ and $\mathcal{V}$.

Given a uniform space $(X, \mathcal{U})$ and a set $M \subseteq X$, the family

$$
\mathcal{U}_{M}=\{(M \times M) \cap V: V \in \mathcal{U}\} \subseteq \mathcal{D}_{M}
$$

is a uniformity on the set $M$ and $\left(M, \mathcal{U}_{M}\right)$ is called a uniform subspace of $X$. Moreover, the topology induced on $M$ by the uniformity $\mathcal{U}_{M}$ coincides with the topology of the subspace $M$ of $X$, where $X$ has the topology induced by $\mathcal{U}$.

Let $\left\{\left(X_{s}, \mathcal{U}_{s}\right): s \in S\right\}$ be a family of uniform spaces and let $X=\prod\left\{X_{s}: s \in S\right\}$. The family of all entourages of the diagonal $\Delta \subseteq X \times X$ which are of the form

$$
\left\{\left(\left\{x_{s}\right\},\left\{y_{s}\right\}\right) \in X \times X:\left|x_{s_{i}}-y_{s_{i}}\right|<V_{i} \text { for } i=1,2, \ldots, n\right\},
$$

where $s_{1}, s_{2}, \ldots, s_{n} \in S$ and $V_{i} \in \mathcal{U}_{s_{i}}$ for $i=1,2, \ldots, n$, is a base for a uniformity called the Cartesian product of the uniformities $\left\{\mathcal{U}_{s}: s \in S\right\}$ which is denoted by $\prod\left\{\mathcal{U}_{s}: s \in S\right\}$. The uniform space $\left(X, \prod\left\{\mathcal{U}_{s}: s \in S\right\}\right)$ is called the Cartesian product of the uniform spaces $\left\{\left(X_{s}, \mathcal{U}_{s}\right): s \in S\right\}$. Moreover, the topology induced on $X$ by the uniformity $\prod\left\{\mathcal{U}_{s}: s \in S\right\}$ coincides with the Tychonoff topology of the Cartesian product $X$, where $X_{s}$ has the topology induced by $\mathcal{U}_{s}$, and each projection map $p_{s}$ from $X$ onto $X_{s}$ is uniformly continuous with respect to $\prod\left\{\mathcal{U}_{s}: s \in S\right\}$ and $\mathcal{U}_{s}$. 
An inverse system of uniform spaces is a family $S=\left\{\left(X_{\alpha}, \mathcal{U}_{\alpha}\right), f_{\alpha}^{\beta}, \Lambda\right\}$, where $\left\{X_{\alpha}, f_{\alpha}^{\beta}, \Lambda\right\}$ is an inverse system of sets and for any $\alpha, \beta \in \Lambda$ satisfying $\alpha \leq \beta$ the mapping $f_{\alpha}^{\beta}$ is uniformly continuous with respect to $\mathcal{U}_{\beta}$ and $\mathcal{U}_{\alpha}$. The uniform space $\left(X_{\Lambda}, \mathcal{U}\right)$, where $X_{\Lambda}=\lim _{\longleftarrow}\left\{X_{\alpha}, f_{\alpha}^{\beta}, \Lambda\right\}$ and $\mathcal{U}=\left(\prod\left\{\mathcal{U}_{\alpha}: \alpha \in \Lambda\right\}\right)_{X_{\Lambda}}$, is called the inverse limit of the inverse system $S$ and is denoted by $\lim S$.

\section{2. $\mathcal{P}$-Like COMPACTA}

In this section we define a $U$-map between uniform spaces and a $\mathcal{P}$-like uniform space. We also extend some classical results related to $\varepsilon$-maps and $\mathcal{P}$-like metric spaces.

Definition 2.1. A mapping $f:(X, \mathcal{U}) \rightarrow(Y, \mathcal{V})$ is a $U$-map, where $U \in \mathcal{U}$, provided that $\delta\left(f^{-1}(y)\right)<U$ for each $y \in Y$.

Theorem $2.2([5,2.7])$. Let $f:(X, \mathcal{U}) \rightarrow(Y, \mathcal{V})$ be a $U$-map between compact uniform spaces. Then there exists $V \in \mathcal{V}$ such that $\delta\left(f^{-1}[B]\right)<U$ whenever $\delta(B)<V$.

Corollary $2.3([5,2.8])$. Let $f:(X, \mathcal{U}) \rightarrow(Y, \mathcal{V})$ be a $U$-map between compact uniform spaces. Then there exists $V \in \mathcal{V}$ such that $|f(x)-f(y)| \geq V$ whenever $|x-y| \geq U$.

Definition 2.4. Let $\mathcal{P}$ be a family of uniform spaces. A uniform space $(X, \mathcal{U})$ is $\mathcal{P}$-like provided that for each $U \in \mathcal{U}$ there exist $(Y, \mathcal{V}) \in \mathcal{P}$ and a surjective $U$-map $f:(X, \mathcal{U}) \rightarrow(Y, \mathcal{V})$. If $\mathcal{P}=\{(Y, \mathcal{V})\}$, we say that $(X, \mathcal{U})$ is $(Y, \mathcal{V})$-like (see [5, 2.9]).

Lemma 2.5. Let $(X, \mathcal{U})$ be a compact $\mathcal{P}$-like uniform space. If every uniform space $(Y, \mathcal{V}) \in \mathcal{P}$ is $\mathcal{Q}$-like, then $(X, \mathcal{U})$ is $\mathcal{Q}$-like.

Proof. Let $U \in \mathcal{U}$ and let $f:(X, \mathcal{U}) \rightarrow(Y, \mathcal{V})$ be a surjective $U$-map, where $(Y, \mathcal{V}) \in \mathcal{P}$. By Theorem 2.2, there exists $V \in \mathcal{V}$ such that $\delta\left(f^{-1}[B]\right)<U$ whenever $\delta(B)<V$. Since $(Y, \mathcal{V})$ is $\mathcal{Q}$-like, there exists a surjective $V$-map $g:(Y, \mathcal{V}) \rightarrow$ $(Z, \mathcal{W})$, where $(Z, \mathcal{W}) \in \mathcal{Q}$. It is not difficult to see that $g \circ f:(X, \mathcal{U}) \rightarrow(Z, \mathcal{W})$ is a $U$-map. Therefore, $(X, \mathcal{U})$ is $\mathcal{Q}$-like.

Lemma 2.6. The inverse limit of an inverse system $S=\left\{\left(X_{\alpha}, \mathcal{U}_{\alpha}\right), f_{\alpha}^{\beta}, \Lambda\right\}$ of compact uniform spaces with surjective bonding maps is $\left\{\left(X_{\alpha}, \mathcal{U}_{\alpha}\right): \alpha \in \Lambda\right\}$-like.

Proof. Let $X_{\Lambda}=\lim _{\longleftarrow} S$ and let $\mathcal{U}=\left(\prod\left\{\mathcal{U}_{\alpha}: \alpha \in \Lambda\right\}\right)_{X_{\Lambda}}$. Let

$$
V=\left\{\left(\left\{x_{\alpha}\right\},\left\{y_{\alpha}\right\}\right):\left|x_{\alpha_{i}}-y_{\alpha_{i}}\right|<V_{i} \text { for } i=1,2, \ldots, n\right\} \in \prod\left\{\mathcal{U}_{\alpha}: \alpha \in \Lambda\right\} .
$$

Let $\alpha_{0} \in \Lambda$ be such that $\alpha_{i} \leq \alpha_{0}$ for each $i \in\{1,2, \ldots, n\}$. It is clear that the projection map $p_{\alpha_{0}} \mid X_{\Lambda}$ is uniformly continuous with respect to $\mathcal{U}$ and $\mathcal{U}_{\alpha_{0}}$. Moreover, by [1, 3.2.15], the map $\left.p_{\alpha_{0}}\right|_{X_{\Lambda}}$ is onto.

Let $a \in X_{\alpha_{0}}$ and let $\left\{x_{\alpha}\right\},\left\{y_{\alpha}\right\} \in\left(p_{\alpha_{0}} \mid X_{\Lambda}\right)^{-1}(a)$. Since $x_{\alpha_{0}}=y_{\alpha_{0}}=a$, we have that $x_{\alpha_{i}}=f_{\alpha_{i}}^{\alpha_{0}}\left(x_{\alpha_{0}}\right)=f_{\alpha_{i}}^{\alpha_{0}}\left(y_{\alpha_{0}}\right)=y_{\alpha_{i}}$ for each $i \in\{1,2, \ldots, n\}$. Then $\left|\left\{x_{\alpha}\right\}-\left\{y_{\alpha}\right\}\right|<V$ which shows that $\left.p_{\alpha_{0}}\right|_{X_{\Lambda}}$ is a $V$-map. Therefore $X_{\Lambda}$ is $\left\{\left(X_{\alpha}, \mathcal{U}_{\alpha}\right): \alpha \in \Lambda\right\}$-like.

Definition 2.7. A continuum is a Hausdorff compact connected topological space.

As a consequence of [5, 2.10] and the last result, we have: 
Theorem 2.8. The inverse limit of arcs with surjective bonding maps is a chainable continuum.

From Lemmas 2.5 and 2.6, we obtain:

Theorem 2.9. Let $X_{\Lambda}$ be the inverse limit of an inverse system $\left\{\left(X_{\alpha}, \mathcal{U}_{\alpha}\right), f_{\alpha}^{\beta}, \Lambda\right\}$ of compact uniform spaces with surjective bonding maps. If every $\left(X_{\alpha}, \mathcal{U}_{\alpha}\right)$ is $\mathcal{P}$-like, then $X_{\Lambda}$ is $\mathcal{P}$-like.

As a consequence of [5, 2.10] and the last result, we have:

Theorem 2.10. The inverse limit of chainable continua is a chainable continuum.

\section{THE SPAN AND UNIVERSAL MAPPINGS}

In [3] and [4], A. Lelek introduced the concepts of the span, the surjective span, the semispan and the surjective semispan of metric spaces. In [2, K. P. Hart and B. J. van der Steeg defined six types of span zero for non-metrizable continua and they prove that chainable continua have surjective semispan zero and that the continua $\mathbb{H}^{*}$ and $\mathbb{I}_{u}$ are nonchainable and have span nonzero. Recently, in [5], the author defined the surjective semispan for Hausdorff continua. In this section we define the span, the surjective span and the semispan of a Hausdorff continuum and we characterize the emptiness of these notions in terms of universal mappings.

Let $\pi_{1}$ and $\pi_{2}$ denote the projection maps from $X \times Y$ onto $X$ and $Y$, respectively.

In [5], the author introduced the following concept:

Definition 3.1. Let $\mathcal{U}$ be a uniformity on a continuum $X$. The surjective semispan of $X$ is the set:

$$
\begin{gathered}
\sigma_{0}^{*}(X)=\left\{V \in \mathcal{U}: \text { there exists a continuum } Z_{V} \subseteq X \times X\right. \text { such that } \\
\left.\pi_{1}\left[Z_{V}\right]=X \text { and } Z_{V} \cap V=\emptyset\right\} .
\end{gathered}
$$

Now, we give other definitions:

Definition 3.2. Let $\mathcal{U}$ be a uniformity on a continuum $X$.

(a) The semispan of $X$ is the set:

$\sigma_{0}(X)=\left\{V \in \mathcal{U}\right.$ : there exists a continuum $Z_{V} \subseteq X \times X$ such that $\pi_{2}\left[Z_{V}\right] \subseteq \pi_{1}\left[Z_{V}\right]$ and $\left.Z_{V} \cap V=\emptyset\right\}$.

(b) The surjective span of $X$ is the set:

$$
\begin{gathered}
\sigma^{*}(X)=\left\{V \in \mathcal{U}: \text { there exists a continuum } Z_{V} \subseteq X \times X\right. \text { such that } \\
\left.\pi_{1}\left[Z_{V}\right]=\pi_{2}\left[Z_{V}\right]=X \text { and } Z_{V} \cap V=\emptyset\right\} .
\end{gathered}
$$

(c) The span of $X$ is the set:

$$
\begin{gathered}
\sigma(X)=\left\{V \in \mathcal{U}: \text { there exists a continuum } Z_{V} \subseteq X \times X\right. \text { such that } \\
\left.\pi_{1}\left[Z_{V}\right]=\pi_{2}\left[Z_{V}\right] \text { and } Z_{V} \cap V=\emptyset\right\} .
\end{gathered}
$$

From the previous definitions, we obtain that:

and

$$
\sigma^{*}(X) \subseteq \sigma_{0}^{*}(X) \subseteq \sigma_{0}(X)
$$

$$
\sigma^{*}(X) \subseteq \sigma(X) \subseteq \sigma_{0}(X)
$$

We also obtain from the previous definition the following result:

Theorem 3.3. Let $\mathcal{U}$ be a uniformity on a continuum $X$, let $Y$ be a subcontinuum of $X$ and let $\gamma \in\left\{\sigma_{0}, \sigma\right\}$. If $(Y \times Y) \cap V \in \gamma(Y)$, then $V \in \gamma(X)$. 
Corollary 3.4. Let $Y$ be a subcontinuum of $X$ and let $\gamma \in\left\{\sigma_{0}, \sigma\right\}$. If $\gamma(X)=\emptyset$, then $\gamma(Y)=\emptyset$.

In Theorem 3.7 we will prove that the emptiness of the surjective semispan, the semispan, the surjective span and the span are preserved by monotone mappings.

Definition 3.5. A continuous mapping $f: X \rightarrow Y$ is monotone provided that $f^{-1}(y)$ is connected for every $y \in Y$.

It is not difficult to see that $f \times f$ is monotone when $f$ is.

Lemma 3.6. Let $\mathcal{U}$ and $\mathcal{V}$ be uniformities on the continua $X$ and $Y$, respectively, let $f: X \rightarrow Y$ be an onto monotone mapping and let $\gamma \in\left\{\sigma_{0}^{*}, \sigma_{0}, \sigma^{*}, \sigma\right\}$. If $V \in \gamma(Y)$, then there exists $U \in \gamma(X)$.

Proof. Suppose that $V \in \sigma_{0}^{*}(Y)$. Then there exists a continuum $Z_{V} \subseteq Y \times Y$ such that $\pi_{1}\left[Z_{V}\right]=Y$ and $Z_{V} \cap V=\emptyset$. By Theorem 1.5, the mapping $f$ is uniformly continuous. Then there exists $U \in \mathcal{U}$ such that $|f(x)-f(y)|<V$ whenever $|x-y|<U$. Then the compactum $Z_{U}=(f \times f)^{-1}\left[Z_{V}\right] \subseteq X \times X$ does not intersect $U$. Let $x \in X$. Since $\pi_{1}\left[Z_{V}\right]=Y$ and $f$ is onto, there exists $y \in X$ such that $(f(x), f(y)) \in Z_{V}$. Then $x \in \pi_{1}\left[Z_{U}\right]$ since $(x, y) \in Z_{U}$. Hence, $\pi_{1}\left[Z_{U}\right]=X$. In order to prove that $U \in \sigma_{0}^{*}(X)$, it suffices to see that $Z_{U}$ is connected, which follows from [1, 6.1.29].

Similar arguments apply for $V \in \sigma_{0}(Y) \cup \sigma^{*}(Y) \cup \sigma(Y)$.

As a consequence of the last result, we have:

Theorem 3.7. Let $f: X \rightarrow Y$ be an onto monotone mapping between continua and let $\gamma \in\left\{\sigma_{0}^{*}, \sigma_{0}, \sigma^{*}, \sigma\right\}$. If $\gamma(X)=\emptyset$, then $\gamma(Y)=\emptyset$.

Using the ideas to prove 3.2 in [5], we obtain:

Theorem 3.8. (a) The semispan of a continuum $X$ is empty if and only if every subcontinuum $Z$ of $X \times X$ satisfying $\pi_{2}[Z] \subseteq \pi_{1}[Z]$ intersects the diagonal $\Delta$ of $X$.

(b) The surjective span of a continuum $X$ is empty if and only if every subcontinuum $Z$ of $X \times X$ satisfying $\pi_{1}[Z]=\pi_{2}[Z]=X$ intersects the diagonal $\Delta$ of $X$.

(c) The span of a continuum $X$ is empty if and only if every subcontinuum $Z$ of $X \times X$ satisfying $\pi_{1}[Z]=\pi_{2}[Z]$ intersects the diagonal $\Delta$ of $X$.

From the last theorem, it follows that in the metric case, the semispan (resp. the surjective span, the span), defined by Lelek in [4, is zero if and only if the semispan (resp. the surjective span, the span) we have defined is empty. This also holds if we use the definition given by Hart and van der Steeg in [2].

Lemma 3.9. Let $\mathcal{U}$ be a uniformity on a continuum $X$ and let $V \in \mathcal{U}$.

(a) $V \in \sigma_{0}^{*}(X)$ if and only if there exist a continuum $A$ and a pair of continuous mappings $f, g: A \rightarrow X$ such that $f[A]=X$ and $(f(a), g(a)) \notin V$ for every $a \in A$.

(b) $V \in \sigma_{0}(X)$ if and only if there exist a continuum $A$ and a pair of continuous mappings $f, g: A \rightarrow X$ such that $g[A] \subseteq f[A]$ and $(f(a), g(a)) \notin V$ for every $a \in A$. 
(c) $V \in \sigma^{*}(X)$ if and only if there exist a continuum $A$ and a pair of continuous mappings $f, g: A \rightarrow X$ such that $f[A]=g[A]=X$ and $(f(a), g(a)) \notin V$ for every $a \in A$.

(d) $V \in \sigma(X)$ if and only if there exist a continuum $A$ and a pair of continuous mappings $f, g: A \rightarrow X$ such that $f[A]=g[A]$ and $(f(a), g(a)) \notin V$ for every $a \in A$.

Proof. (a) Suppose $V \in \sigma_{0}^{*}(X)$. Then there exists a continuum $Z_{V} \subseteq X \times X$ such that $\pi_{1}\left[Z_{V}\right]=X$ and $Z_{V} \cap V=\emptyset$. Thus, the continuum $A=Z_{V}$ and the continuous mappings $f=\pi_{1}$ and $g=\pi_{2}$ satisfy the conclusion.

In order to prove the other implication, suppose that a continuum $A$ and a pair of continuous mappings $f, g: A \rightarrow X$ satisfy $f[A]=X$ and $(f(a), g(a)) \notin V$ for every $a \in A$. Then the continuum

$$
Z_{V}=\{(f(a), g(a)): a \in A\} \subseteq X \times X
$$

satisfies $\pi_{1}\left[Z_{V}\right]=X$ and $Z_{V} \cap V=\emptyset$. Thus, $V \in \sigma_{0}^{*}(X)$.

Similar arguments prove (b), (c) and (d).

Lemma 3.10. Let $X$ be a continuum.

(a) $\sigma_{0}^{*}(X)=\emptyset$ if and only if for any continuum $A$ and a pair of continuous mappings $f, g: A \rightarrow X$ satisfying $f[A]=X$ there exists $a \in A$ such that $f(a)=g(a)$.

(b) $\sigma_{0}(X)=\emptyset$ if and only if for any continuum $A$ and a pair of continuous mappings $f, g: A \rightarrow X$ satisfying $g[A] \subseteq f[A]$ there exists $a \in A$ such that $f(a)=g(a)$.

(c) $\sigma^{*}(X)=\emptyset$ if and only if for any continuum $A$ and a pair of continuous mappings $f, g: A \rightarrow X$ satisfying $f[A]=g[A]=X$ there exists $a \in A$ such that $f(a)=g(a)$.

(d) $\sigma(X)=\emptyset$ if and only if for any continuum $A$ and a pair of continuous mappings $f, g: A \rightarrow X$ satisfying $f[A]=g[A]$ there exists $a \in A$ such that $f(a)=g(a)$.

Proof. (a) Suppose that a continuum $A$ and a pair of continuous mappings $f, g$ : $A \rightarrow X$ satisfy $f[A]=X$ and $f(a) \neq g(a)$ for every $a \in A$. Then the continuum

$$
Z_{V}=\{(f(a), g(a)): a \in A\} \subseteq X \times X
$$

satisfies $\pi_{1}\left[Z_{V}\right]=X$ and $Z_{V} \cap \Delta=\emptyset$. Thus, by [5, 3.2], $\sigma_{0}^{*}(X) \neq \emptyset$.

The other implication is a consequence of Lemma 3.9 (a).

Similar arguments prove (b), (c) and (d), using Theorem 3.8 and Lemma 3.9.

Definition 3.11. A space $X$ has the fixed point property provided that for every continuous mapping $f: X \rightarrow X$ there exists $x \in X$ such that $f(x)=x$.

Definition 3.12. A continuous mapping $f: X \rightarrow Y$ is universal if for every continuous mapping $g: X \rightarrow Y$ there exists $x \in X$ such that $f(x)=g(x)$.

It is not difficult to see that if $f: X \rightarrow Y$ is universal, then $f$ is surjective and $Y$ has the fixed point property.

Definition 3.13. A surjective continuous mapping $f: X \rightarrow Y$ is universal with respect to surjective maps if for every surjective continuous mapping $g: X \rightarrow Y$ there exists $x \in X$ such that $f(x)=g(x)$. 
As a consequence of Lemma 3.10, we have:

Theorem 3.14. $\quad$ (a) A continuum $X$ has empty surjective semispan if and only if every continuous mapping $f: A \rightarrow X$ from a continuum $A$ onto $X$ is universal.

(b) A continuum $X$ has empty semispan if and only if every continuous mapping $f: A \rightarrow X$ from a continuum $A$ into $X$ satisfies that $f: A \rightarrow f[A]$ is universal.

(c) A continuum $X$ has empty surjective span if and only if every continuous mapping $f: A \rightarrow X$ from a continuum $A$ onto $X$ is universal with respect to surjective maps.

(d) A continuum $X$ has empty span if and only if every continuous mapping $f: A \rightarrow X$ from a continuum $A$ into $X$ satisfies that $f: A \rightarrow f[A]$ is universal with respect to surjective maps.

The remark following Definition 3.12 and the last result yield:

Corollary 3.15. $\quad$ (a) Continua with empty surjective semispan have the fixed point property.

(b) Each subcontinuum of a continuum with empty semispan has the fixed point property.

Theorem 3.16. $\quad$ (a) A continuum has empty semispan if and only if each of its subcontinua has empty surjective semispan.

(b) A continuum has empty span if and only if each of its subcontinua has empty surjective span.

Proof. (a) Suppose that a continuum $X$ has empty semispan. Let $Y$ be a subcontinuum of $X$ and let $f: A \rightarrow Y$ be a continuous mapping from a continuum $A$ onto $Y$. By Theorem 3.14 (b), $f: A \rightarrow Y \subseteq X$ satisfies that $f: A \rightarrow f[A]=Y$ is universal. Then, by Theorem 3.14 (a), $Y$ has empty surjective semispan. Now, suppose that each subcontinuum of $X$ has empty surjective semispan. Let $f: A \rightarrow X$ be a continuous mapping from a continuum $A$ into $X$. Since $f[A]$ is a subcontinumm of $X$, by Theorem 3.14 (a), $f: A \rightarrow f[A]$ is universal, then, by Theorem 3.14 (b), $X$ has empty semispan.

Similar arguments prove (b) using Theorem 3.14 (c) and Theorem 3.14(d).

The proof of [5, 3.6] can be applied to prove the following two results:

Lemma 3.17. Let $\gamma \in\left\{\sigma_{0}^{*}, \sigma^{*}\right\}$ and let $f:(X, \mathcal{U}) \rightarrow(Y, \mathcal{V})$ be a surjective $U$-map between continua. If $U \in \gamma(X)$ and $V \in \mathcal{V}$ is as in Corollary 2.3, then $V \in \gamma(Y)$.

Proof. Suppose that $U \in \sigma_{0}^{*}(X)$ and let $Z_{U}$ be a subcontinuum of $X \times X$ such that $\pi_{1}\left[Z_{U}\right]=X$ and $Z_{U} \cap U=\emptyset$. Let $V \in \mathcal{V}$ be as in Corollary 2.3.

We consider the subcontinuum

$$
Z=\left\{(f(x), f(y)):(x, y) \in Z_{U}\right\}
$$

of $Y \times Y$. By Corollary 2.3, we know that $|f(x)-f(y)| \geq V$ for each $(x, y) \in Z_{U}$. Hence, $V \in \sigma_{0}^{*}(Y)$.

Similar arguments apply for $\gamma=\sigma^{*}$.

Lemma 3.18. Let $\gamma \in\left\{\sigma_{0}, \sigma\right\}$ and let $f:(X, \mathcal{U}) \rightarrow(Y, \mathcal{V})$ be a $U$-map between continua. If $U \in \gamma(X)$ and $V \in \mathcal{V}$ is as in Corollary 2.3, then $V \in \gamma(Y)$. 
The last two lemmas imply the following:

Theorem 3.19. Let $\mathcal{P}$ be a family of continua and let $\gamma \in\left\{\sigma_{0}^{*}, \sigma_{0}, \sigma^{*}, \sigma\right\}$. If a continuum $(X, \mathcal{U})$ is $\mathcal{P}$-like and $\gamma(Y)=\emptyset$ for every $(Y, \mathcal{V}) \in \mathcal{P}$, then $\gamma(X)=\emptyset$.

From [5, 2.10] and the last result, we have:

Theorem 3.20. Each chainable continuum has $\gamma(X)=\emptyset$ for $\gamma \in\left\{\sigma_{0}^{*}, \sigma_{0}, \sigma^{*}, \sigma\right\}$.

As a consequence of Lemma 2.6 and Theorem 3.19, we obtain:

Theorem 3.21. Let $S=\left\{\left(X_{\alpha}, \mathcal{U}_{\alpha}\right), f_{\alpha}^{\beta}, \Lambda\right\}$ be an inverse system of continua with surjective bonding maps and let $X_{\Lambda}$ be its inverse limit. If $\gamma \in\left\{\sigma_{0}^{*}, \sigma_{0}, \sigma^{*}, \sigma\right\}$ and $\gamma\left(X_{\alpha}\right)=\emptyset$ for every $\alpha \in \Lambda$, then $\gamma\left(X_{\Lambda}\right)=\emptyset$.

\section{ACKNOWLEDGEMENT}

The author thanks the referee for comments and suggestions.

\section{REFERENCES}

1. R. Engelking, General topology, Heldermann Verlag, Berlin, 1989. MR.1039321 (91c:54001)

2. K. P. Hart, B. J. van der Steeg, Span, chainability and the continua $\mathbb{H}^{*}$ and $\mathbb{I}_{u}$, Topology Appl., 151(2005), 226-237. MR2139754 (2006m:54063)

3. A. Lelek, Disjoint mappings and the span of spaces, Fund. Math., 55(1964), 199-214. MR0179766 (31:4009)

4. A. Lelek, On the surjective span and semispan of connected metric spaces, Colloq. Math., 37(1977), 35-45. MR0482680 (58:2737)

5. A. Peláez, The surjective semispan for Hausdorff continua, to appear in Topology and its Applications.

Universidad Autónoma de la Ciudad de México, Plantel Cuautepec, Avenida la Corona 320, Gustavo A. Madero, C.P. 07160, México D. F.

E-mail address: pelaez@matem.unam.mx 